July 2001

\title{
Mindfulness-based stress reduction and health-related quality of life in a heterogeneous patient population
}

\author{
Diane K. Reibel \\ Thomas Jefferson University \\ Jeffrey M. Greeson \\ Thomas Jefferson University \\ George C. Brainard \\ Thomas Jefferson University \\ Steven Rosenzweig MD \\ Thomas Jefferson University
}

Follow this and additional works at: https://jdc.jefferson.edu/jmbcimfp

Part of the Alternative and Complementary Medicine Commons

Let us know how access to this document benefits you

\section{Recommended Citation}

Reibel, Diane K.; Greeson, Jeffrey M.; Brainard, George C.; and Rosenzweig, Steven MD, "Mindfulness-based stress reduction and health-related quality of life in a heterogeneous patient population" (2001). Marcus Institute of Integrative Health Faculty Papers. Paper 2.

https://jdc.jefferson.edu/jmbcimfp/2

This Article is brought to you for free and open access by the Jefferson Digital Commons. The Jefferson Digital Commons is a service of Thomas Jefferson University's Center for Teaching and Learning (CTL). The Commons is a showcase for Jefferson books and journals, peer-reviewed scholarly publications, unique historical collections from the University archives, and teaching tools. The Jefferson Digital Commons allows researchers and interested readers anywhere in the world to learn about and keep up to date with Jefferson scholarship. This article has been accepted for inclusion in Marcus Institute of Integrative Health Faculty Papers by an authorized administrator of the Jefferson Digital Commons. For more information, please contact: JeffersonDigitalCommons@jefferson.edu. 


\title{
Mindfulness-based stress reduction and health-related quality of life in a heterogeneous patient population
}

\section{Diane K. Reibel Ph.D.,* Jeffrey M. Greeson M.S., George C. Brainard Ph.D., and Steven Rosenzweig M.D.}

\author{
Center for Integrative Medicine, Thomas Jefferson University, Philadelphia, PA, USA
}

*Corresponding author. Tel.: +1-215-955-1376; fax: +1-215-503-0414; email: diane.reibel@mail.tju.edu. This study was supported in part by the Advanta Corporation, Goldsmith-Greenfield Foundation, and Jefferson Medical College.

\begin{abstract}
This study examined the effects of mindfulness-based stress reduction (MBSR) on health-related quality of life and physical and psychological symptomatology in a heterogeneous patient population. Patients $(n=136)$ participated in an 8 -week MBSR program and were required to practice 20 min of meditation daily. Pre- and postintervention data were collected by using the Short-Form Health Survey (SF-36), Medical Symptom Checklist (MSCL) and Symptom Checklist-90 Revised (SCL-90-R). Healthrelated quality of life was enhanced as demonstrated by improvement on all indices of the SF-36, including vitality, bodily pain, role limitations caused by physical health, and social functioning (all $P<.01$ ). Alleviation of physical symptoms was revealed by a $28 \%$ reduction on the MSCL $(P<.0001)$. Decreased psychological distress was indicated on the SCL-90-R by a 38\% reduction on the Global Severity Index, a 44\% reduction on the anxiety subscale, and a $34 \%$ reduction on the depression subscale (all $P<.0001$ ). Oneyear follow-up revealed maintenance of initial improvements on several outcome parameters. We conclude that a group mindfulness meditation training program can enhance functional status and well-being and reduce physical symptoms and psychological distress in a heterogeneous patient population and that the intervention may have long-term beneficial effects.
\end{abstract}

Keywords: Stress; Mindfulness; Meditation; Health-related quality of life

\section{Introduction}

It is estimated that over 100 million Americans live with chronic illness [1]. Many face tremendous challenges with disruption of their day-to-day physical, social, and vocational functioning [2, 3 and 4]. Furthermore, there is a high prevalence of physical and psychological distress among the chronic illness population, including pain; anxiety; depression; and feelings of isolation, hopelessness, and helplessness [4 and 5]. In addition to the high cost of chronic illness on patients' personal lives, the financial cost to the health care system is also very high. In 1990, the projected direct costs of medical care for patients with chronic illness ran approximately US $\$ 425$ billion, $61 \%$ of the nation's health care expenditures for the year [1]. As the average age of our population increases and chronic illness rates expand, it is imperative to develop and implement low-cost, 
effective, therapeutic interventions that help alleviate suffering and improve patient functionality.

Mindfulness-based stress reduction (MBSR), founded by Jon Kabat-Zinn, PhD, in 1979, is designed to teach patients with chronic medical conditions how to live fuller, healthier, more adaptive lives [6]. MBSR is a clinical group intervention that is patient-centered, experiential, and educational. The core of the program involves intensive training in mindfulness meditation and its applications for daily living and coping with stress, pain, and illness. Mindfulness meditation is moment-to-moment awareness that is intentionally non-reactive and non-judgmental. The practitioner attends to the full range of whatever is present in the field of his or her experience in a non-judgmental way. This makes mindfulness a highly practical inner orientation for people facing illness and its accompanying emotional turbulence [7]. Mindfulness meditation helps in facing all aspects of life, however painful, with increasing degrees of equanimity, wisdom, and compassion. It empowers the practitioner with the ability to respond consciously rather than to react automatically to events. These qualities are cultivated as the practitioner spends formal time each day in periods of silence with the focus on present moment experience, and then as the practitioner carries the moment-to-moment awareness into various aspects of daily living.

Results of initial research on the effectiveness of MBSR in various patient populations have been very promising. Kabat-Zinn et al. [8] found that chronic pain patients trained in a 10-week MBSR program showed significant reductions in present moment pain, inhibition of daily activity by pain, and pain-related drug use. Participants in that study also experienced a reduction in anxiety, depression, and self-reported medical symptoms. A follow-up study showed sustained improvement in all parameters up to 4 years, with the exception of present moment pain [9]. In another clinical study, patients with anxiety disorders based on DSM-III-R diagnoses experienced significant reductions in anxiety and panic following an 8-week MBSR program [10]. Maintenance of these improvements was retained up to 3 years following the intervention [11]. Kaplan et al. [12] examined the effects of a 10-week MBSR program in patients with fibromyalgia and found improvement on scales of pain, sleep, fatigue, and global well-being.

More recently, a bilingual MBSR program was offered to a heterogeneous, inner-city patient population [13]. Participants experienced a reduction in medical and psychological symptoms, as well as an increase in self-esteem. In this study, patients also reported dramatic changes in attitudes, habits, and behaviors that might ultimately improve health directly and/or increase compliance with proven health care regimens.

The present investigation was designed to examine the effects of MBSR on health-related quality of life as well as physical and psychological symptoms in a heterogeneous patient population. MBSR was found to enhance patients' functional status and well-being and to reduce physical symptoms and psychological distress.

\section{Methods}


A prospective, observational clinical trial with open enrollment was conducted between 1997 and 1999. Twelve separate MBSR courses were offered during this time period. Each course ran for 8 weeks and consisted of weekly, 2.5-h group sessions and a full day (6-7 h) intensive meditation retreat held in the sixth week of the program. During the weekly sessions a variety of forms of mindfulness meditation were taught and practiced, including body scan, awareness of breathing, mindful hatha yoga, eating meditation, walking meditation, and guided imagery (mountain/lake meditations). Class time each week was divided between meditation practice, small and large group discussions, and mindfulness skill-building activities. Class activities were designed to (1) enhance awareness of one's body and mind, (2) teach participants to replace automatic reactions with consciously chosen responses, and (3) bring greater awareness and skill to interpersonal communication. Group discussions focused on participants' experiences with meditation practices and on the applications of mindfulness in day-to-day life. Participants received audiocassette tapes for daily meditation practice and were asked to practice 20 min of formal meditation a day, 6 days per week.

Participants met with the course instructor for a one-on-one entrance interview prior to starting the program. Medical history and informed consent were obtained during this meeting. During the interview, prospective enrollees were given a detailed description of the program, including a strong emphasis on the required commitment for daily meditation practice.

Several instruments were used to assess physical and mental health status across the intervention period. Three health-related questionnaires, including the Medical Outcomes Study Short-Form Health Survey (SF-36), Symptom Checklist-90 Revised (SCL-90-R), and Medical Symptom Checklist (MSCL), were administered before and after the program. The SF-36 is a 36-item survey that reports health-related quality of life, including both physical and mental functioning and well-being [14 and 15]. This instrument is widely used in the evaluation of allopathic treatment modalities and includes one multi-item scale measurement for each of eight health concepts: (1) physical functioning (PF); (2) role-physical (RP), role limitations because of physical health problems; (3) bodily pain (BP); (4) general health (GH) perception; (5) vitality (VT), energy/fatigue; (6) social functioning (SF); (7) role-emotional (RE), role limitations because of emotional problems; and (8) mental health $(\mathrm{MH})$, psychological distress and psychological well-being [14]. Each scale is scored from 0 to 100, the highest functional status level being 100. Physical Component Summary (PCS) and Mental Component Summary (MCS) scores also can be obtained by grouping the four scales most relevant to physical health status (PF, RP, BP, GH) and the four scales most relevant to mental health status (VT, SF, RE, MH), respectively. In addition to providing scale scores, the two summary measures can be interpreted in the form of a percentile relative to the general population [16].

The MSCL was used to quantify the number of medical symptoms reported for the preceding month [17]. Over-all psychological distress, anxiety, depression, and somatization were assessed by using the SCL-90-R, a 90-item inventory that assesses the presence and severity of somatic and psychological symptoms on a scale of 0 to 4 [18]. 
The summary score, Global Severity Index (GSI) as well as the anxiety (ANX), depression (DEP), and somatization (SOM) subscales combine information on the number of symptoms reported and the intensity of perceived distress for each symptom.

In addition to pre- and post-course health surveys, participants also completed 10-point Likert-scale ratings of body tension, mental clarity, and well-being before and after each weekly session. These pre- and post-class ratings were used to quantify changes that occurred during a single session of mindfulness-based stress reduction.

Furthermore, a questionnaire was administered during the first class meeting to assess participants' expectancy regarding the efficacy of the MBSR program. Exit surveys were administered at the conclusion of the eighth and final session. Information obtained in the exit survey included adherence to meditation practice, post-treatment ability to cope with stress, post-treatment change in sense of well-being, and overall satisfaction with the course.

Paired Student's $t$ tests were performed to compare pre- and post-intervention scores on each health-related instrument, as well as on pre- and post-class session data. To control for multiple comparisons and to minimize the Type I error rate, a Bonferroni correction was employed. Only differences of $P<.005$ and $P<.013$ were considered significant on the SF-36 and SCL-90-R, respectively. Trend level differences $(P<.05)$ were noted for descriptive purposes only. Effect sizes were calculated for each outcome measure administered before and after the program to assess the degree of clinical relevance, as well as to facilitate comparisons with existing and future research studies. Analysis of variance (ANOVA) with repeated measures was performed to assess long-term changes in health-related quality of life and medical and psychological symptoms. Post hoc tests, including Fisher's PLSD and Sheffe's $F$ test, were used to further examine relationships among pre-intervention, post-intervention, and 1-year follow-up scores on dependent variables. In addition, a cluster analysis was performed to examine within-group effects

of separate MBSR courses on health-related outcome measures [19]. All data are reported as mean \pm SEM unless otherwise indicated. Alpha was set at 05 .

\section{Results}

\section{Baseline characteristics of study population}

The study sample ( $n=136)$ was composed of patients with heterogeneous medical diagnoses. Subjects' ages ranged from 23 to 76 years (Mean=47.2 \pm 1.0 years). Ninety-six participants (71\%) were women; $40(29 \%)$ were men. One hundred twenty-one individuals (89\%) were Caucasian, $13(10 \%)$ were African-American, and two (1\%) were biracial. Sixty-two study participants $(46 \%)$ were married, 31 (23\%) were single, 26 $(19 \%)$ were separated or divorced, and six $(4 \%)$ were widowed (marital status was not available for 11 subjects). At intake, 91 participants $(67 \%)$ were employed, $17(13 \%)$ were unemployed, nine (7\%) were retired, and four (3\%) were students (occupational status was not available for 15 subjects). Seventy-six participants (56\%) reported their 
current work status as "active," whereas 28 individuals $(21 \%)$ stated that they were presently "disabled" (work status was not available for 32 subjects).

The most prevalent medical conditions reported by patients during initial screening interviews were chronic pain $(n=37)$, hypertension $(n=28)$, and anxiety/panic disorder $(n=24)$, although the range of reported diagnoses was broad (Table 1). One-third $(33 \%)$ of the study participants reported having only one primary medical condition, while twothirds $(67 \%)$ reported having two or more major illnesses. Specifically, 35 patients $(27 \%)$ had two conditions, 25 (19\%) had three conditions, 17 (13\%) had four conditions, and 11 $(8 \%)$ had more than four conditions. These data reflect significant comorbidity in the present patient population.

\section{Table 1}

Frequency and range of primary and secondary medical conditions reported by study participants in a meditation-based stress reduction program

\begin{tabular}{lr} 
Medical Condition & Total Number $^{\mathrm{a}}$ \\
\hline AIDS/HIV & \\
Amputation, Arm & 4 \\
Angina & 1 \\
Anxiety/Panic disorder & 2 \\
Arthritis/Tendonitis & 24 \\
Asthma/Allergies & 9 \\
Cancer & 14 \\
Cerebral hemorrhage & 16 \\
Depression & 1 \\
Diabetes & 22 \\
Dizziness, Chronic & 2 \\
Epilepsy & 1 \\
Fatigue & 2 \\
Fibromyalgia & 4 \\
Gastrointestinal distressb & 6 \\
Headaches & 12 \\
Heart disease & 8 \\
Herniated disc & 3 \\
Hypertension & 2 \\
Irregular heartbeat & 28 \\
Lupus erythematosus & 3 \\
Multiple sclerosis & 1 \\
Obsessive-Compulsive disorder & 3 \\
Pain, Chronic & 2 \\
Peripheral neuropathy & 37 \\
Seasonal affective disorder & 1 \\
Sickle Cell Anemia & 1 \\
Sinusitis & 1 \\
Sleep disorders & 5 \\
Stress & 10 \\
& 15
\end{tabular}


${ }^{\text {a }}$ Total number exceeds 136 because of comorbidity among patients.

${ }^{\text {b} C r o h n ' s ~ d i s e a s e ~(1), ~ G a s t r o e n t e r i t i s ~(1), ~ G a s t r o e s o p h a g e a l ~ r e f l u x ~ d i s e a s e ~}$

(3), Irritable bowel syndrome (1), Peptic ulcer disease (1), undiagnosed

stomach distress (5).

Table 2

Pre-intervention SF-36 scores for MBSR study population $(n=100-104)$ vs. the general U.S. adult population $(n=2474)$

\begin{tabular}{|c|c|c|c|c|c|c|c|c|c|c|}
\hline Sample & $\mathrm{PF}$ & $\mathrm{RP}$ & $\mathrm{BP}$ & $\mathrm{GH}$ & VT & SF & $\mathrm{RE}$ & MH & MCS & PCS \\
\hline MBSR Patients & $76.2^{\mathrm{a}}$ & $52.2^{\mathrm{a}}$ & $61.7^{\mathrm{a}}$ & $59.1^{\mathrm{a}}$ & $43.1^{\mathrm{b}}$ & $67.5^{\mathrm{b}}$ & $64.9^{\mathrm{b}}$ & $58.0^{\mathrm{b}}$ & $42^{\mathrm{b}}$ & $45^{\mathrm{b}}$ \\
\hline U.S. Adult Norm & 84.1 & 81.0 & 75.1 & 71.9 & 60.9 & 83.3 & 81.3 & 74.7 & 52 & 53 \\
\hline U.S. Adult 25th Percentile & 70.0 & 50.0 & 61.0 & 57.0 & 45.0 & 75.0 & 66.7 & 64.0 & 45 & 46 \\
\hline
\end{tabular}

${ }^{\mathrm{a}}$ Score below general U.S. population norm.

${ }^{\mathrm{b}}$ Score below 25 th percentile of U.S. general population norm [16].

Pre-intervention assessment indicated that the study group scored well below the general U.S. population norm on all eight indexes as well as the two summary scales of the SF-36 (Table 2). Furthermore, it was revealed that the study group scored below the 25th percentile on the four scales most relevant to mental health (VT, SF, RE, MH). In addition, the study sample placed above the 80th percentile on the GSI as well as the ANX, DEP, SOM subscales of the SCL-90-R relative to a normative sample [16] of the general U.S. population (GSI raw score, $n=103 ;=0.72 \pm 0.05$, ANX raw $\mathrm{SCORE}=0.80 \pm 0.05, \mathrm{DEP}$ raw $\mathrm{SCORE}=1.01 \pm 0.07, \mathrm{SOM}$ raw $\mathrm{SCORE}=0.69 \pm 0.06)$.

A brief survey was administered during the first class session in eight of the 12 program cycles to assess subjects' attitudes regarding the anticipated efficacy of the MBSR program. All 90 respondents expected that doing the meditation-based stress reduction practices over the next 8 weeks would reduce their general level of tension. Seventy of 79 subjects $(89 \%)$ anticipated that doing the stress reduction exercises over the next 8 weeks would reduce their medical symptoms. Seventy of 78 subjects with bodily pain $(90 \%)$ anticipated that doing the meditation practices over the next 8 weeks would reduce their pain and/or discomfort. Similarly, 56 of $71(79 \%)$ respondents to the pre-intervention expectation survey anticipated that doing the stress reduction practices over the next 8 weeks would reduce any limitations they may have had because of pain or discomfort.

\section{Outcome measures and statistical analyses}

Of 136 study participants, $121(89 \%)$ completed an 8-week intervention program in mindfulness meditation training. Completion of the program was defined as having attended six or more of the weekly sessions. Five individuals dropped the course because of medical complications unrelated to their participation in the study. Pre- and postintervention data were available for 104 program participants.

Paired Student's $t$ tests were used to compare pre- and post-intervention scores on all outcome instruments, including the SF-36, MSCL, and SCL-90-R. Analyses performed on pre- and post-treatment scores for each of the eight SF-36 subscales revealed 
significant improvements across the study period on all indices (Fig. 1). Whereas the study group placed below the 25 th percentile on all four SF-36 subscales most relevant to mental health on entering the study, following completion of the 8-week program it scored above the 25 th percentile on these measures. This improvement was reflected in the MCS score, which rose from $42.00 \pm 1.16$ to $48.38 \pm 1.00$ ( $t=-6.05 ; d f=97 ; P<.0001$; Fig. 2). This change represented a shift from the 20th to the 33 rd percentile versus the general U.S. population [16]. A lesser, though significant, improvement was also evident on the group Physical Component Summary score, which rose approximately from $45.35 \pm 1.30$ to $47.26 \pm 1.19(t=-2.65 ; d f=97 ; P<.01 ;$ Fig. 2$)$. This change represented a shift from the 24th to the 28th percentile versus the general U.S. population [16].

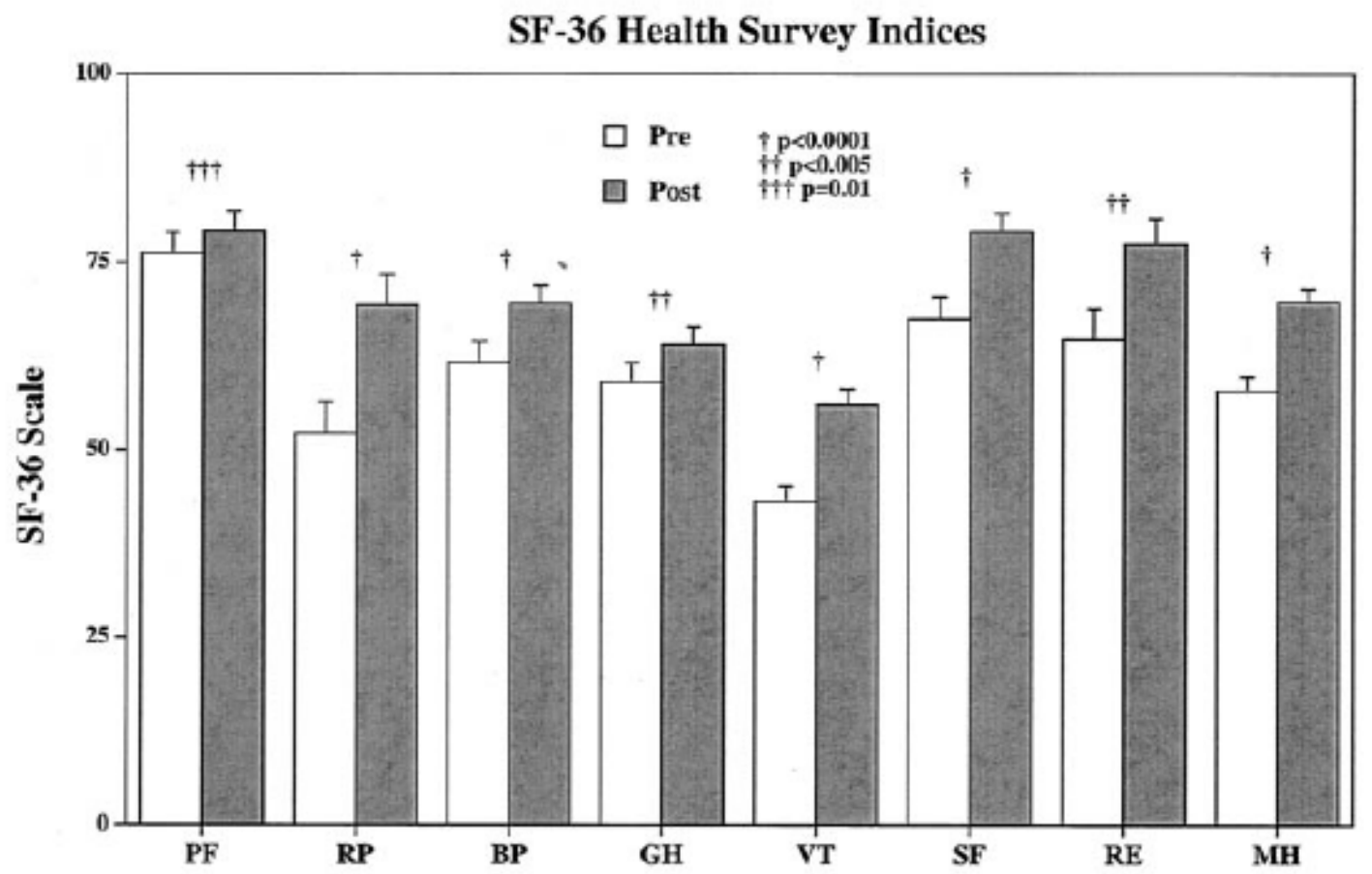

Fig. 1

Pre- and post-intervention scale scores (Mean \pm SEM) on the SF-36 Health Survey ( $n=100-104)$. All indices except $\mathrm{PF}$ are significantly improved at the adjusted level of statistical probability (Bonferroni $\mathrm{p}<0.005)$. PF $=$ Physical Functioning; $\mathrm{RP}=$ Role-Physical; $\mathrm{BP}=$ Bodily Pain; $\mathrm{GH}=$ General Health; VT $=$ Vitality; SF $=$ Social Functioning; RE $=$ Role-Emotional; $\mathrm{MH}=$ Mental Health. 


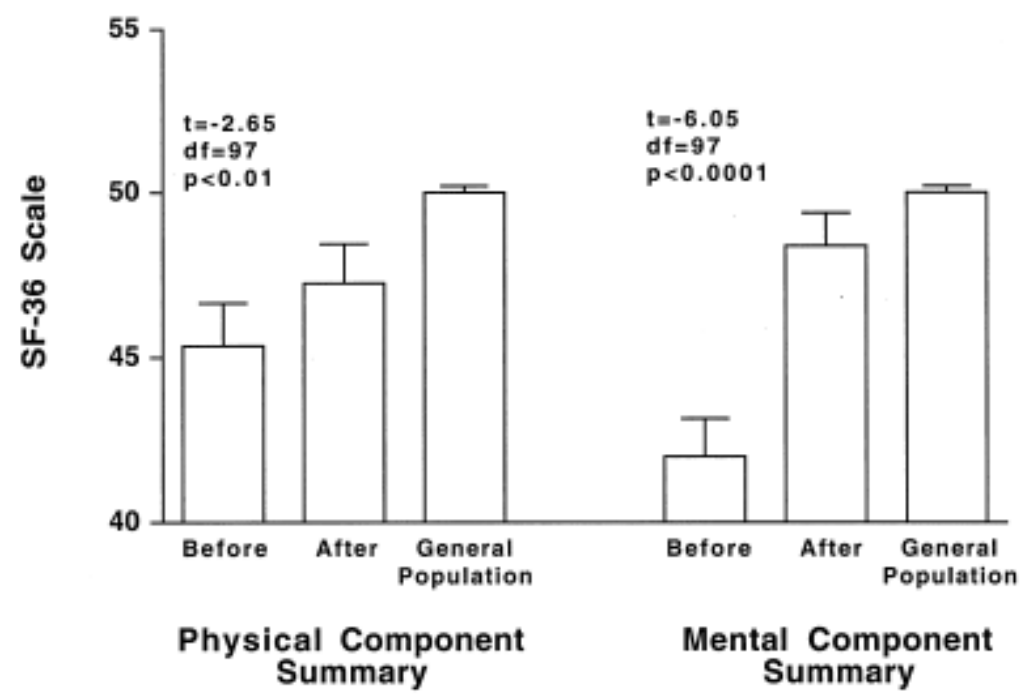

Fig. 2

Pre- and post-intervention physical and mental component summary scores (Mean \pm SEM) on the SF-36 Health Survey $(n=98)$. Post-intervention scores improved significantly and approach general population norms [16] for healthy adults $(n=2,474)$.

Evidence of physical and psychological symptom reduction over the intervention period was demonstrated on the MSCL and SCL-90-R, respectively. The group MSCL score decreased $28 \%$, from $20 \pm 1$ to $14 \pm 1$, a significant reduction in the number of self-reported physical complaints $(t=6.46 ; d f=102 ; P<.0001$; Fig. 3$)$. The group GSI score of the SCL90 -R dropped $38 \%$, from $0.72 \pm 0.05$ to $0.45 \pm 0.04(t=7.10 ; d f=102 ; P<.0001)$. Similar reductions were observed on the ANX (44\%), DEP (34\%), and SOM (25\%) subscales of the SCL-90-R, as group scores improved from $0.80 \pm 0.07$ to $0.45 \pm 0.05,1.01 \pm 0.07$ to $0.67 \pm 0.07$, and $0.69 \pm 0.06$ to $0.52 \pm 0.05$, respectively (ANX: $t=6.47, d f=102, P<.0001$; DEP: $t=6.25, d f=102, P<.0001$; SOM: $t=4.84, d f=102, P<.0001$; Fig. 4$)$. These results indicate a quantitative decrease in psychological and stress-related symptomatology across the 8-week intervention period. 


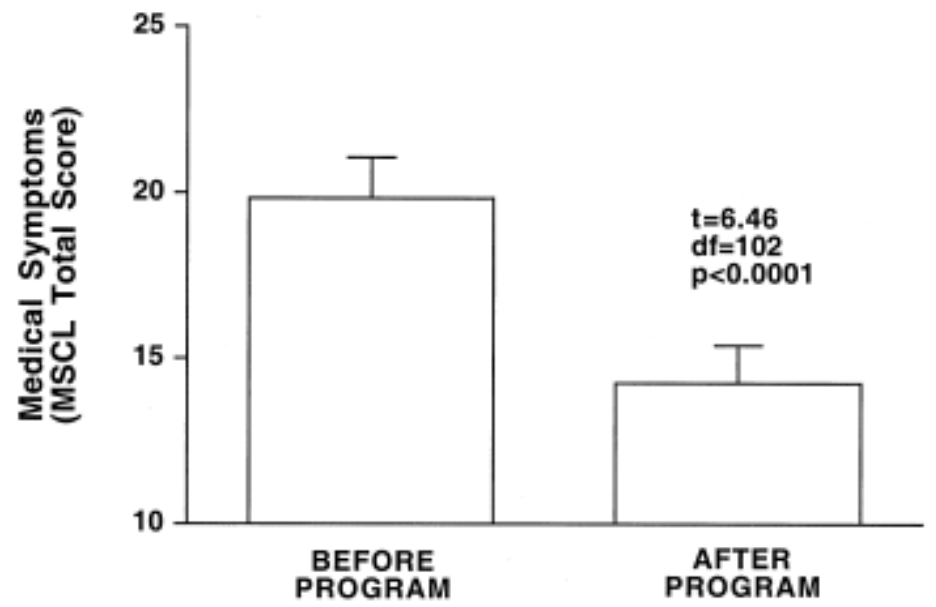

Fig. 3

Pre-treatment versus post-treatment scores (Mean \pm SEM) on the Medical Symptom Checklist (MSCL). These data provide evidence of a significant decline in self-reported medical symptoms across the 8 -week intervention period.

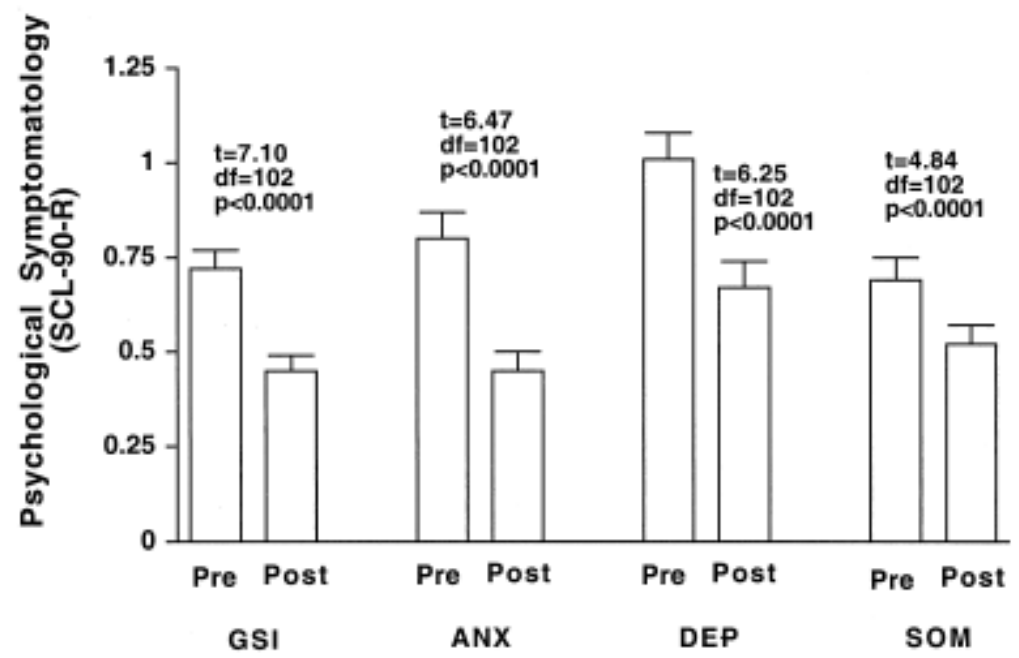

Fig. 4

Pre-treatment versus post-treatment scores (Mean \pm SEM) on the Symptom Checklist-90Revised (SCL-90-R). These data provide evidence of a significant reduction in overall psychological distress, anxiety, depression and somatization across the 8-week intervention period (Bonferroni $\mathrm{p}<0.013$ ). GSI $=$ Global Severity Index, ANX = Anxiety Subscale, DEP $=$ Depression Subscale, $\mathrm{SOM}=$ Somatization Subscale.

Because the MBSR intervention was offered to 12 separate groups of patients, we conducted additional analyses to examine potential effects of intra-group correlation. 
Specifically, we conducted two mixed-model ANOVA on the SCL-90-R GSI, the SCL90-R ANX subscale and the SF-36 MCS score. The first analysis used participant as a random effect, replicating the paired $t$ analysis. The second added group as a random effect. The between-group variance component for all three endpoints was small relative to the between-participant variance components, and consequently the impact of inference was nil. The standard errors from the two analyses agreed to more than four decimal places.

To address the clinical relevance of pre- to post-intervention changes on dependent outcome measures, effect sizes were calculated by dividing the change score for a given index by the SD of the group scores on that index. Effect sizes are generally categorized as small (less than 0.30 SD units), moderate (0.50-0.79 SD units), and large (0.80 SD units or greater) [20]. Table 3 illustrates the effect sizes for all indices that comprise the SF-36 Health Survey, the MSCL, and the SCL-90-R. Small to moderate effects were observed on the SF-36, whereas moderate effects were found on the MSCL and SCL-90R. SF-36 indexes that displayed moderate effect sizes included RP, VT, RE, MH, and MCS. In addition, six of eight SF-36 subscales (RP, BP, VT, SF, RE, MH) improved by five points or more, which is indicative of a clinically or socially relevant change [14]. A seventh index $(\mathrm{GH})$ improved by nearly five points.

Table 3

Effect sizes of clinically or socially relevant changes for matched pre- and postintervention scores on the SF-36, MSCL, and SCL-90-R

\begin{tabular}{|c|c|c|c|c|c|c|}
\hline $\begin{array}{l}\text { Instrument } \\
\text { Scale }\end{array}$ & $n$ & $\begin{array}{l}\text { Pre } \\
\text { Score }\end{array}$ & $\begin{array}{l}\text { Post } \\
\text { Score }\end{array}$ & Difference & $\begin{array}{c}\% \\
\text { Improvement }\end{array}$ & $\begin{array}{l}\text { Effect } \\
\text { Size }^{\mathrm{a}}\end{array}$ \\
\hline \multicolumn{7}{|l|}{$\overline{S F-36}$} \\
\hline PF & 104 & 76.25 & 79.17 & 2.92 & 4 & 0.11 \\
\hline $\mathrm{RP}$ & 102 & 52.21 & 69.36 & 17.15 & 33 & 0.41 \\
\hline BP & 104 & 61.67 & 69.51 & 7.84 & 13 & 0.29 \\
\hline GH & 100 & 59.10 & 63.99 & 4.89 & 8 & 0.20 \\
\hline VT & 104 & 43.14 & 56.03 & 12.89 & 30 & 0.62 \\
\hline SF & 104 & 67.55 & 79.21 & 11.66 & 17 & 0.45 \\
\hline $\mathrm{RE}$ & 102 & 64.87 & 77.46 & 12.59 & 19 & 0.33 \\
\hline MH & 104 & 57.97 & 69.81 & 11.84 & 20 & 0.66 \\
\hline MCS & 98 & 42.00 & 48.38 & 6.38 & 15 & 0.60 \\
\hline PCS & 98 & 45.35 & 47.26 & 1.91 & 4 & 0.15 \\
\hline \multicolumn{7}{|l|}{ MSCL } \\
\hline Total Score & 103 & 20 & 14 & 6 & 30 & 0.49 \\
\hline \multicolumn{7}{|l|}{$S C L-90-R$} \\
\hline GSI & 103 & 0.72 & 0.45 & 0.27 & 38 & 0.55 \\
\hline ANX & 103 & 0.80 & 0.45 & 0.35 & 44 & 0.57 \\
\hline DEP & 103 & 1.01 & 0.67 & 0.34 & 34 & 0.47 \\
\hline SOM & 103 & 0.69 & 0.52 & 0.17 & 25 & 0.30 \\
\hline
\end{tabular}

${ }^{a}$ Effect size calculated as the (Difference)/(average STDEV of MBSR population pre and post scores). An effect size of .50 or greater is considered moderate by conventional standards (Cohen, 1988). Bold 5 moderate effect size, and/or greater than five-point improvement on SF-36 scale. 
Follow-up surveys, which consisted of an SF-36, MSCL, SCL-90-R, and meditation practice assessment form, were administered 1 year following completion of each MBSR course. In patients $(n=41)$ for whom all data were available, ANOVA with repeated measures revealed significant improvements on (1) three of eight SF-36 subscales (VT, SF, MH), (2) the MCS scale of the SF-36, (3) the MSCL, and (4) the GSI and ANX, DEP, and SOM subscales of the SCL-90-R (Table 4). A trend toward significance was observed for several other SF-36 indexes, including RP, BP, and GH perception (Table 4). Post hoc testing for differences between pre-intervention scores and 1-year follow-up scores revealed that numerous outcome parameters (PF, GH, VT, SF, MH, MCS, GSI, ANX, DEP, and SOM) improved significantly according to Fisher's PLSD and Sheffe's $F$ test. Post hoc testing further revealed no significant differences between postintervention and 1-year follow-up scores. Thirty-one of $44(70 \%)$ total respondents to the 1 year follow-up survey indicated that they still practiced formal meditation more than three times per week for 10-20 min per day. Additionally, 40 one-year survey respondents $(91 \%)$ indicated that they practiced meditation informally approximately five times per week.

Table 4.

Pre-intervention, post-intervention, and 1-year follow-up scores on the SF-36, MSCL, and SCL-9-R for a subgroup of MBSR study participants $(n=41)$

\begin{tabular}{|c|c|c|c|c|c|}
\hline Index & $\begin{array}{l}\text { Pre Mean } \\
\text { (SEM) }\end{array}$ & $\begin{array}{l}\text { Post Mean } \\
\text { (SEM) }\end{array}$ & $\begin{array}{l}\text { 1-year } \\
\text { Mean } \\
(\text { SEM) }\end{array}$ & $\begin{array}{l}F \text { test } \\
(2,80)\end{array}$ & $P$-value \\
\hline \multicolumn{6}{|l|}{$S F-36$} \\
\hline $\mathrm{PF}$ & $82.98(3.51)$ & $84.05(3.51)$ & $81.59(4.02)$ & 0.76 & .47 \\
\hline $\mathrm{RP}$ & $57.93(6.62)$ & $73.78(5.65)$ & $72.56(6.34)$ & 4.84 & .01 \\
\hline BP & $65.71(4.46)$ & $72.41(3.80)$ & $67.00(4.27)$ & 3.26 & .04 \\
\hline $\mathrm{GH}$ & $61.85(3.63)$ & $67.36(3.50)$ & $67.93(3.54)$ & 3.95 & .02 \\
\hline VT & $45.24(3.07)$ & $57.20(3.44)$ & $53.66(3.49)$ & 8.37 & $.0005^{\mathrm{a}}$ \\
\hline SF & $69.82(4.23)$ & $81.71(3.24)$ & $78.66(3.47)$ & 5.64 & $.005^{\mathrm{a}}$ \\
\hline $\mathrm{RE}$ & $71.95(6.06)$ & $82.94(4.37)$ & $75.61(5.34)$ & 1.76 & .18 \\
\hline MH & $59.22(2.95)$ & $71.80(2.61)$ & $70.34(2.46)$ & 14.47 & $.0001^{\mathrm{a}}$ \\
\hline MCS & $42.61(1.82)$ & $49.22(1.54)$ & $47.60(1.33)$ & 8.41 & $.0005^{\mathrm{a}}$ \\
\hline PCS & $46.98(1.92)$ & $48.36(1.84)$ & $47.68(2.02)$ & 0.88 & .42 \\
\hline$M S C L$ & $19(2)$ & $13(2)$ & $13(2)$ & 12.57 & $.0001^{\mathrm{a}}$ \\
\hline \multicolumn{6}{|l|}{$S C L-90-R$} \\
\hline GSI & $0.66(0.08)$ & $0.39(0.06)$ & $0.43(0.07)$ & 11.47 & $.0001^{\mathrm{a}}$ \\
\hline ANX & $0.71(0.10)$ & $0.36(0.07)$ & $0.40(0.09)$ & 10.63 & $.0001^{\mathrm{a}}$ \\
\hline DEP & $0.90(0.11)$ & $0.59(0.10)$ & $0.66(0.10)$ & 5.76 & $.005^{\mathrm{a}}$ \\
\hline SOM & $0.63(0.09)$ & $0.49(0.09)$ & $0.50(0.09)$ & 3.83 & .03 \\
\hline
\end{tabular}

${ }^{2}$ Significant change based on repeated measures ANOVA (Bonferroni alpha for SF-36 5 0.005; Bonferroni alpha for SCL-90-R 5 0.013 ). All significant changes occurred between pre- and post-intervention as determined by post hoc testing. There were no significant differences between post-intervention and 1-year follow-up values for any measure. 
In addition to pre- and post-intervention health-related outcomes, analyses were conducted to determine whether changes in body tension, mental clarity, and/or sense of well-being occurred within a single session of mindfulness-based stress reduction training. Paired $t$ tests of pre- and post-class scores from the first class meeting revealed a significant reduction in body tension concomitant with an increase in mental clarity and greater sense of well-being (Fig. 5; body tension: $t=-11.84, d f=94, P<.0001$; mental clarity: $t=-9.04, d f=94, P<.0001$; well-being: $t=-10.09, d f=94, P<.0001)$. Furthermore, it was revealed that pre- and post-class ratings for body tension, mental clarity, and wellbeing were significantly improved in the eighth class session compared to the first class session (all $P<.005)$.

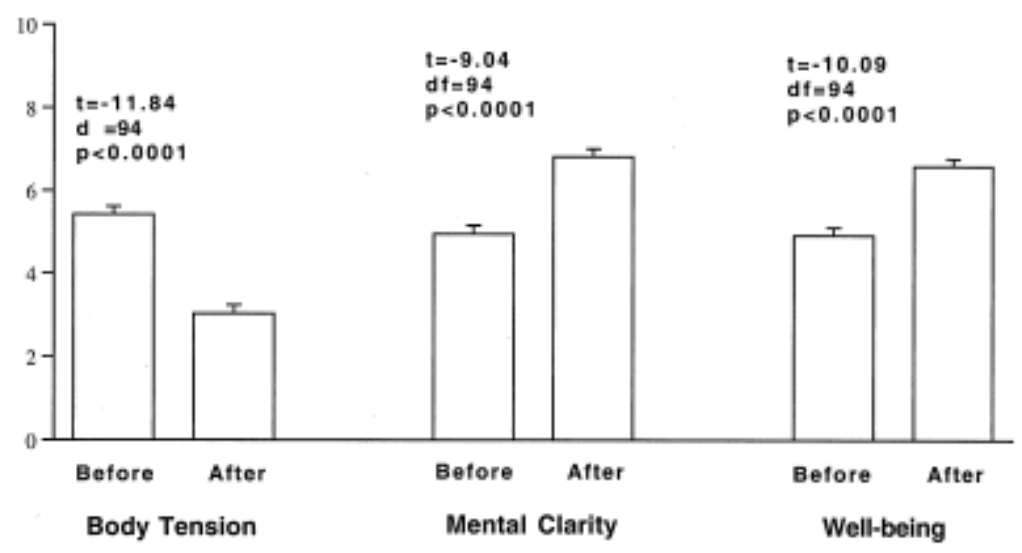

Fig. 5

Pre- versus post-class ratings (Mean \pm SEM) of body tension, mental clarity and wellbeing for the first of eight class sessions in Mindfulness-Based Stress Reduction. Score of $10=$ extremely high, clear, or greatest sense of well-being, respectively. Significantly lower body tension, and greater mental clarity and well-being were reported after the first training session $(n=95)$.

\section{Compliance and patient-rated efficacy of the mindfulness-based stress reduction program}

All individuals who completed the program were asked to return an exit survey, which was administered immediately following the last treatment session. Sixty-four of 112 respondents $(57 \%)$ reported practicing the techniques taught in the course almost every day, as instructed. One hundred one of the study participants $(90 \%)$ reported practicing three times per week or more. Ninety-four of 112 respondents $(84 \%)$ indicated that they practiced for a duration of 15-30 min per session, demonstrating a high level of adherence to our intended 20-min practice time.

When subjects were asked to rate their ability to cope with stress "now compared to before the program" on a 5-point scale, where 1 denoted much worse than before and 5 denoted much better/great improvement, 108 of 111 respondents $(97 \%)$ responded 4 or 5 $(\mathrm{Mean}=4.51 \pm 0.05)$. When asked to rate their sense of well-being "now compared to 
before the program," where 1 denoted much worse than before and 5 denoted much better/great improvement, 106 of 111 respondents $(95 \%)$ answered 4 or 5 (Mean= $4.43 \pm 0.05)$.

In addition, when participants were asked to rate their individual satisfaction with the course on a 5-point scale, where 1 represented very dissatisfied and 5 represented very

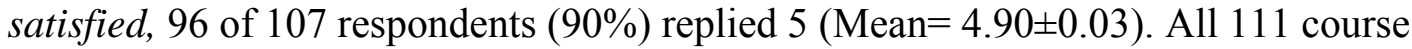
survey respondents indicated that they gained something of lasting value or importance from the MBSR program.

\section{Discussion}

The results of this prospective, observational study demonstrate significantly enhanced health-related quality of life, reduced physical symptoms, and decreased psychological distress in a heterogeneous patient population following an 8-week training program in MBSR. These findings are noteworthy given that the study patients reported a high degree of comorbidity and scored well below the general population norm on all standardize health outcome instruments before the MBSR intervention. Nearly $90 \%$ of the study participants completed the MBSR program, which is consistent with the high rate of program completion reported by researchers at the University of Massachusetts Medical Center [21].

Specifically, significant improvements following the MBSR intervention were observed on all indexes of the SF-36, including VT, BP, SF, and RP. These findings demonstrate enhanced health-related quality of life in terms of patient functionality and well-being. Clinically significant effects were found on six out of eight SF-36 subscales. Significant improvements in self-reported medical symptoms were documented by using the Medical Symptom Checklist. Significant reduction in psychological distress, including anxiety and depression, was demonstrated by changes in the SCL-90-R. Again, these changes on the MSCL and SCL-90-R represent clinically significant effect sizes. One-year follow-up data available for a subset of the patient population revealed maintenance of the majority of initial improvements in health-related quality of life, medical symptoms, and psychological distress. The present investigation supports the hypothesis that patients suffering from various health problems can enhance their daily functioning and wellbeing and alleviate physical and psychological symptoms by participating in an intensive 8-week MBSR program. In addition, at least some participants in the program can experience long-term beneficial effects.

Previous research conducted by Kabat-Zinn et al. [8] revealed that following training in MBSR patients with chronic pain experienced a decrease in (1) self-reported medical symptoms; (2) anxiety, depression, and over-all psychological distress; and (3) bodily pain. Similar improvements in medical symptoms and psychological parameters were reported in patients with anxiety disorders who participated in an MBSR program [10]. Interestingly, comparable changes in medical symptoms and psychological distress were attained in our heterogeneous patient population that practiced mindfulness meditation formally for $20 \mathrm{~min}$ a day, as compared to the 45 -minute daily practice implemented by 
Kabat-Zinn. It is important to note, however, that unmeasured outcomes may be different between patients who practice 20 versus 45 min of meditation daily.

Long-term follow-up surveys have demonstrated maintenance of initial improvements in physical and psychological symptoms among patients with chronic pain [9] and anxiety disorders [11] trained in MBSR. Our 1-year follow-up data also indicate maintenance of initial improvements on several health-related outcome measures, including VT, selfreported medical symptoms, over-all psychological distress, anxiety, and depression. However, a potential confound to the long-term health promotion effects observed in our follow-up study is that only $30 \%$ of MBSR program participants responded to the 1-year follow-up survey. It is possible that this group may have been composed largely of individuals who experienced improvement in their health status during the program and subsequently maintained their meditation practice. Individuals who may not have experienced a noticeable change in their physical or mental functioning or well-being, and/or did not continue their meditation practice beyond the duration of the 8-week course, may not have been inclined to return the follow-up survey.

Additionally, it is possible that the long-term therapeutic effects observed in a subset of our patient population may not have been caused exclusively by continued formal meditation practice. Although $70 \%$ of respondents to the 1-year follow-up survey indicated that they practiced formal meditation three times per week for 10-20 min per session, a higher proportion (91\%) of respondents noted that they practiced informal meditation more frequently, at five times per week. Informal mindfulness meditation practice involves moment-to-moment awareness in the course of daily activity, enabling one to respond versus to react to life events. In this way, informal mindfulness practice promotes psychological resilience and resistance to stress through cognitive restructuring. As described previously by Kabat-Zinn et al. [10], such changes in cognitive appraisals help to reduce anxiety and promote one's ability to cope with future stressful situations more effectively. Thus, the insight and increased range of options associated with an enhanced feeling of control may account, in part, for the long-term improvements in psychological and physical health observed in our 1-year follow-up cohort. Despite uncertainty regarding the way in which positive health changes are maintained following mindfulness meditation training, we may conclude that MBSR is associated with longterm beneficial effects in at least a subgroup of participants.

In addition to potential long-term health promotion effects, our study suggests that MBSR practice is associated with changes in body tension, mental clarity, and sense of well-being as early as the first class session. Further, our data indicate that body tension, mental clarity, and well-being improve throughout the training period.

The major limitation of our study, as well as most other clinical investigations of MBSR, is the lack of a control group and randomized patient assignment. To date, only two randomized, controlled studies of MBSR in patient populations have been published. Astin [22] examined the effects of an 8-week MBSR program in chronic pain patients by using a randomized, controlled study design (wait-list controls) and found a significant reduction in psychological symptomatology (depression and anxiety) and an increase in 
perceived control in those patients who participated in MBSR. Patients in the MBSR group also had higher scores on Inspirit, a measure of spiritual experiences. The major limitation of that study was a small sample size (14 participants per group). More recently, Speca et al. [23] demonstrated that MBSR was associated with significantly reduced mood disturbance and symptoms of stress in a heterogeneous group of cancer outpatients. The only other randomized, controlled study, published by Shapiro et al. [24], involved a nonpatient population. Although study participants were healthy medical and premedical students, levels of state and trait anxiety and depression were reduced, whereas empathy increased following MBSR training.

MBSR programs contain several potentially therapeutic elements that may account for observed improvements in physical and mental health among participants. These include mindfulness meditation training as well as other therapeutic factors inherent in the group intervention, such as emotional expression and social support. At present, it is unknown to what extent training in mindfulness meditation alone is responsible for health improvements. Numerous well-controlled studies demonstrated that meditation practice itself can have profound mind/body effects [25, 26, 27, 28 and 29]. In one recent randomized, controlled trial, patients who practiced mindfulness mediation while undergoing ultraviolet light treatments for moderate to severe psoriasis were found to heal at approximately four times the rate of those patients who received ultraviolet treatment alone [28]. In that study, the meditation intervention was delivered by using an audiocassette tape recording in the isolation of a light booth, so social support variables were not present. Rigorous clinical research on a different form of meditation practice (transcendental meditation) has revealed reduced blood pressure and decreased progression of atherosclerosis in hypertensive patients [27 and 29].

MBSR group interventions, in addition to teaching specific meditation techniques, provide participants with a highly supportive group environment that may also promote healing. As part of the MBSR group, individuals have an opportunity to express their feelings about their illness and share their experiences regarding meditation practice. Social support and emotional expression in one's effort to cope with illness are in all likelihood important factors in healing [30]. There is increasing evidence that people with chronic illnesses who come together in support groups to share their common experiences, to gain knowledge about their illness, and/or to learn coping skills show improvements in both psychological and physical symptoms and may possibly prolong their survival time [4, 31, 32, 33 and 34]. Future, controlled trials ought to help differentiate the contributing effects of the various therapeutic elements inherent in MBSR programs.

In addition to the elements of social support and emotional expression, patient expectancy may also contribute to the healing response associated with MBSR. At the outset of our study, $100 \%$ of MBSR program participants anticipated a reduction in their general level of tension following meditation training. Similarly, $80-90 \%$ of study participants expected MBSR to reduce their medical symptoms, bodily pain, and/or limitations because of pain or discomfort. It is well known that patient belief and expectancy can influence medical outcomes [35], and our findings suggest that such an 
effect may contribute to the positive health outcomes associated with MBSR. Controlled trials of MBSR would need to account for this potentially significant variable.

In summary, the results of this observational study demonstrate that an 8-week training program in mindfulness meditation is associated with significant and clinically relevant improvements in health-related quality of life, medical symptoms, and psychological distress among patients with various chronic health problems. In addition, our study suggests that initial improvements in physical and mental functioning and well-being

might be maintained in some MBSR participants up to 1 year following completion of the intervention. Future studies are needed to (1) examine the efficacy of MBSR in larger randomized, controlled trials; and (2) address the potential therapeutic effect of psychosocial support in MBSR programs by comparing MBSR to other supportive group interventions that do not include mindfulness training. At present, the literature suggests that patients with chronic medical conditions who participate in MBSR are able to affect positive changes in their physical and mental health status, presumably the result of an increased ability to cope with stress, pain, and illness. The health promotion effects of MBSR appear to complement conventional biomedical treatment in a comprehensive, patient-centered approach to healing and alleviation of human suffering.

\section{Acknowledgements}

We thank Gerard Santini for his invaluable assistance in the Stress Reduction Program. We thank Carol Rabinowitz and Walter Hauck, Ph.D., for their assistance with data analysis. We give heartfelt appreciation to Jon Kabat-Zinn, Ph.D., and Saki Santorelli, Ed.D., for their ongoing support and guidance.

\section{References}

1. C. Hoffman, D. Rice and H-Y Sung, Persons with chronic conditions. JAMA 276 (1996), pp. 1473-1479.

2. K. Lorig, H. Holman, D. Sobel et al.. Living a Healthy Life with Chronic Conditions: Self Management of Heart Disease, Arthritis, Stroke, Diabetes, Asthma, Bronchitis, Emphysema and Others, Bull Press, Palo Alto (1994).

3. J.C. Parker, K.L. Smarr, S.P. Buckelew, R.C. Stucky-Ropp and J.E. Hewett, Effects of stress management on clinical outcomes in rheumatoid arthritis. Arthritis Rheum 38 (1995), pp. 1807-1818.

4. C.F. Telch and M.J. Telch, Group coping skills instruction and supportive group therapy for cancer patients: a comparison of strategies. J Consult Clin Psychol 54 (1986), pp. 802-808.

5. R.F. Meenan, E.H. Yelin, M. Nevitt and W.V. Epstein, The impact of chronic disease: a socio-medical profile of rheumatoid arthritis. Arthritis Rheum $\mathbf{2 4}$ (1981), pp. 544-548.

6. J. Kabat-Zinn. Full Catastrophe Living: Using the Wisdom of Your Body and Mind to Face Stress, Pain and Illness, Delacorte, New York (1990).

7. J. Kabat-Zinn, Mindfulness Meditation: What It Is, What It Isn't and Its Role in Health Care and Medicine. In: Y. Haruki and M. Suzuki, Editors, Comparative 
and Psychological Study on Meditation, Eburon, Delft, Netherlands (1996), pp. 161-170.

8. J. Kabat-Zinn, L. Lipworth and R. Burney, The clinical use of mindfulness meditation for the self-regulation of chronic pain. J Behav Med 8 (1985), pp. $163-$ 190.

9. J. Kabat-Zinn, L. Lipworth, R. Burney and W. Sellers, Four year follow-up of a meditation-based program for the self-regulation of chronic pain: treatment outcomes and compliance. Clin J Pain 2 (1986), pp. 159-173.

10. J. Kabat-Zinn, A.O. Massion, J. Kristeller et al., Effectiveness of a meditationbased stress reduction intervention in the treatment of anxiety disorders. Am J Psychiatry 149 (1992), pp. 936-943.

11. J. Miller, K. Fletcher and J. Kabat-Zinn, Three-year follow-up and clinical implications of a mindfulness-based stress reduction intervention in the treatment of anxiety disorders. Gen Hosp Psychiatry 17 (1995), pp. 192-200.

12. R.H. Kaplan, D.L. Goldberg and M. Galvin-Nadeau, The impact of a meditationbased stress reduction program on fibromyalgia. Gen Hosp Psychiatry 15 (1993), pp. 284-289.

13. B. Roth and T. Creaser, Mindfulness meditation-based stress reduction: experience with a bilingual inner-city population. Nurse Pract 22 (1997), pp. $150-176$.

14. J.E. Ware. SF-36 Health Survey Manual and Interpretation Guide, The Health Institute, Boston (1993).

15. J.E. Ware and C.D. Sherbourne, The MOS 36-Item Short-Form Health Survey (SF-36). I. Conceptual framework and item selection. Med Care 30 (1992), pp. 473-483.

16. J.E. Ware, M. Kosinski and S.D. Keller. SF-36 Physical and Mental Health Summary Scales: A User's Manual, Health Assessment Lab, Boston (1994).

17. J. Kabat-Zinn, An outpatient program in behavioral medicine for chronic pain patients based on the practice of mindfulness meditation: theoretical considerations and preliminary results. Gen Hosp Psychiatry 4 (1982), pp. 33-47.

18. Derogatis LR. SCL-90-R Administration, Scoring, and Procedures Manual, Third Ed. Minneapolis: National Computer Systems, Inc., 1994.

19. A. Donner, N. Birkett and C. Buck, Randomization by cluster: sample size requirements and analysis. Am J Epidemiol 114 (1981), pp. 906-914.

20. J. Cohen. Statistical Power for the Behavioral Sciences, Lawrence Erlbaum, Hillsdale (1988).

21. J. Kabat-Zinn and A. Chapman-Waldrop, Compliance with an outpatient stress reduction program: rates and predictors of program completion. J Behav Med $\mathbf{1 1}$ (1988), pp. 333-352.

22. J.A. Astin, Stress reduction through mindfulness meditation. Psychother Psychosom 66 (1997), pp. 97-106.

23. M. Speca, L.E. Carlson, E. Goodey and M. Angen, A randomized, wait-list controlled clinical trial: the effect of a mindfulness meditation-based stress reduction program on mood and symptoms of stress in cancer outpatients. Psychosom Med 62 (2000), pp. 613-622. 
24. S.L. Shapiro, G.E. Schwartz and G. Bonner, Effects of mindfulness-based stress reduction on medical and premedical students. J Behav Med 21 (1998), pp. 581599.

25. C.N. Alexander, P. Robinson, D.W. Orme-Johnson, R.H. Schneider and K.G. Walton, The effects of transcendental meditation compared to other methods of relaxation and meditation in reducing risk factors, morbidity, and mortality. Homeostasis 35 (1994), pp. 243-263.

26. H. Benson. The Relaxation Response, William Morrow, New York (1975).

27. A. Castillo-Richmond, R.H. Schneider, C.N. Alexander and R. Cook, Effects of stress reduction on carotid atherosclerosis in hypertensive African-Americans. Stroke 31 (2000), pp. 568-573.

28. J. Kabat-Zinn, E. Wheeler, T. Light et al., Influence of a mindfulness-based stress reduction intervention on rates of skin clearing in patients with moderate to severe psoriasis undergoing phototherapy (UVB) and photochemotherapy (PUVB). Psychosom Med 60 (1998), pp. 625-632.

29. R.H. Schneider, F. Staggers, C.N. Alexander and W. Sheppard, A randomized controlled trial of stress reduction for hypertension in older African-Americans. Hypertension 26 (1996), pp. 820-827.

30. D. Ornish. Love and Survival. The Scientific Basis for the Healing Power of Intimacy, Harper Collins, New York (1998).

31. D.F. Cella, B. Sarafin, P.R. Snider, S.B. Yellen and P. Winicour, Evaluation of a community-based cancer support group. Psychooncology 2 (1993), pp. 123-132.

32. P. Fosbair, Cancer support groups and group therapies, part 1: historical and theoretical backgrounds and research on effectiveness. J Psychosoc Oncol 15 (1997), pp. 63-81.

33. D. Spiegel, J.R. Bloom, H.C. Kraemer and E. Gottheil, Effect of psychosocial treatment on survival of patients with metastatic breast cancer. Lancet 2 (1989), pp. $888-891$.

34. A. Trojan, Benefits of self-help groups: a survey of 232 members from 65 disease-related groups. Soc Sci Med 29 (1999), pp. 225-232.

35. A. Harrington, Editor, The Placebo Effect: An Interdisciplinary Exploration, Harvard University Press, Cambridge (1999). 